\title{
Observables, Disassembled
}

\author{
Bryan W. Roberts \\ Philosophy, Logic 83 Scientific Method \\ Centre for Philosophy of Natural and Social Sciences \\ London School of Economics \& Political Science \\ Houghton Street, London WC2A 2AE, UK \\ b.w.roberts@lse.ac.uk
}

\begin{abstract}
How should we characterise the observable aspects of quantum theory? This paper argues that philosophers and physicists should jettison a standard dogma: that observables must be represented by self-adjoint or Hermitian operators. Four classes of non-standard observables are identified: normal operators, symmetric operators, real-spectrum operators, and none of these. The philosophical and physical implications of each are explored.
\end{abstract}

\section{INTRODUCTION}

There is a disconnect between standard accounts of mathematical representation and standard accounts of physical observables. From the perspective of the philosophy of representation, we enjoy extraordinary freedom in choosing what mathematical objects can represent things. In contrast, most well-developed accounts of observables insist on restricting to a tiny corner of mathematics involving real numbers. Nowhere is this dogma more stark than in quantum mechanics, where observables

Acknowledgements. Thanks to Jeremy Butterfield, Michel Janssen, Casey McCoy, and two anonymous referees for helpful comments and corrections that improved this paper. Any remaining errors are my own. 
are generally associated with the real-number eigenvalues of self-adjoint operators. My aim in this paper is to show how this restriction on quantum observables can be given up, and to identify the important new classes of observables that arise as a consequence.

The restriction to real numbers is sometimes motivated by appeal to an old worry about complex numbers, which should be immediately dispelled. Consider a bead that is constrained to move on a ring. We could represent its position using pairs $(r, \theta)$ of real numbers, or using the complex circle $R e^{i \theta} \in \mathbb{C}$ with $R \in \mathbb{R}^{+}$and $\theta \in$ $[0,2 \pi)$. Of course, there was once considerable scepticism about the status of complex numbers, which led to the use of the word 'imaginary' in describing them. ${ }^{1}$ But such misgivings should not trouble us today: the complex numbers can be constructed axiomatically in just the same sense as the real numbers. So, it is difficult to see a sense in which the two representations are not equally adequate. Viewing the real and the complex circles as embedded in $\mathbb{C}^{2}$, we even find the two are related by a rigid rotation, shown in Figure 1.

Nevertheless, textbook discussions of quantum theory almost always insist that observables must involve real numbers and self-adjoint operators, as in Sakurai's classic treatment: "[w]e expect on physical grounds that an observable has real eigenvalues... That is why we talk about Hermitian observables in quantum mechanics" (Sakurai 1994, §1.3). Similarly, Griffiths writes, "the expectation value of an observable quantity has got to be a real number (after all, it corresponds to actual measurements in the laboratory, using rulers and clocks and meters)" (Griffiths 1995, $\S 3.3)$. And Weinberg writes, "[w]e can now see why it is important for all operators representing observable quantities to be Hermitian. ... Hermitian operators have real

\footnotetext{
${ }^{1}$ Cardano derived complex solutions to the equation $x^{2}-10 x+30=0$ in his 1545 Ars Magna, but concluded, "So progresses arithmetic subtlety the end of which, as is said, is as refined as it is useless" (Cardano 1968, §37). Over 200 years later Euler took a similar view: "they are usually called imaginary quantities, because they exist merely in the imagination", although he argued that "nothing prevents us from making use of these imaginary numbers, and employing them in calculation" (Euler 1822, p.43).
} 


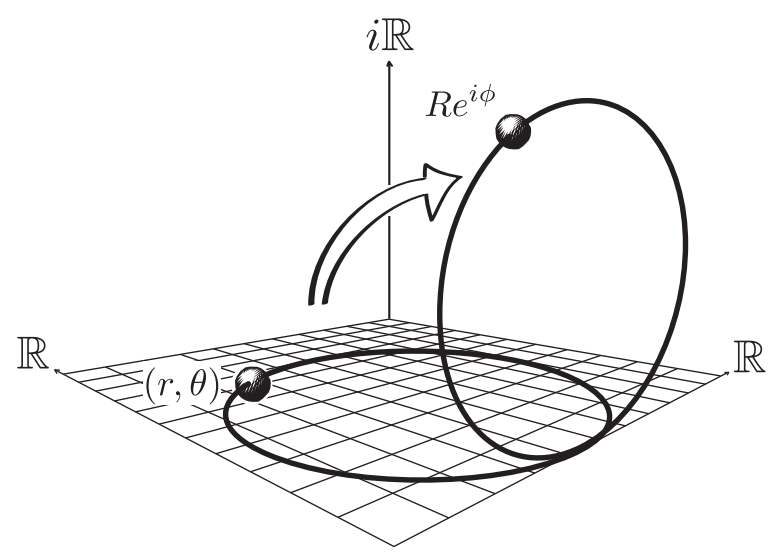

FiguRE 1. Real and complex descriptions of particle position related by a rotation in $\mathbb{C}^{2}$.

expectation values" (Weinberg 2013, p.24). Even when one encounters quantum field operators that are not self-adjoint, such as the free Klein-Gordon field, this is quickly explained away as equivalent to a commuting pair of operators that are self-adjoint.

The philosophy of quantum mechanics has largely followed the textbooks. For example, Hughes writes that self-adjoint operators "represent physical quantities, and their eigenvalues will be the possible values of those quantities; clearly it befits a measurable quantity that its possible values should be real" (Hughes 1992, p.33). Similarly, Albert's book on the philosophy of quantum mechanics sets out what he calls 'principle (B)', that measurable properties are to be represented by linear operators, and then states, "it's clear from principle (B) (since, of course, the values of physically measurable quantities are always real numbers) that the operators associated with measurable properties must necessarily be Hermitian operators" (Albert 1992, p.40). Similar remarks are found in many other places in physics and philosophy.

The thesis of this paper is that the orthodoxy should be given up: there are many physically and philosophically interesting ways to have a non-self-adjoint observable. In particular, the self-adjointness property may be broken down into three 'component' properties: being normal, being symmetric, and having a real spectrum, each defined precisely below. Observables can be represented by non-self-adjoint 
operators that have any one of these properties while giving up the other two, or that give up all three.

The unorthodox observables that I will advocate here have been discussed before. Indeed, we will see that each has been advocated by prominent physicists, and that two in particular are associated with active research programmes: symmetric operators amount to a positive operator-valued measure or 'POVM' approach to quantum observables, while the real-spectrum condition forms the basis for so-called 'PT-symmetric' quantum theory. The 'normal operators' approach is not as wellunderstood, and so I will develop it beyond existing discussions. However, my aim here is not to introduce new physics. Rather, I would like to reduce some of the confusion that philosophical and textbook treatments of observables appear to have introduced. I also aim to clarify the connections between these unorthodox research programmes. It is striking that few physicists advocating one of the non-standard approaches appear to recognise any of the others. I hope this discussion might help improve their mutual recognition, by identifying the role that each plays in the philosophical foundations of observables.

The plan of the paper is as follows. The second section will introduce the dogma of self-adjoint operators, and then propose a way to classify the possible nonself-adjoint observables. The third section considers non-self-adjoint operators that are normal. Here I argue that existing proposals in favour of normal operators must be restricted using the concept of what I call a 'sharp set'. The fourth section explores the physics of non-normal operators. First I consider non-normal operators that are symmetric but do not have a real spectrum; these turn out to amount to a 'POVM' approach to observables, and also allow for the introduction of 'time observables'. Next, I consider operators that have a real spectrum but are not symmetric; these include $P T$-symmetric observables. Finally, I consider operators that do not have any of these three properties: they are not normal, do not have a real spectrum, and are not symmetric. The fifth section is the conclusion. 


\section{Self-Adjointness Disassembled}

2.1. The history of self-adjointness. How did we come to require self-adjoint observables? It began when Heisenberg arrived in Göttingen in June of 1925 with a draft of his celebrated paper on non-commutative mechanics. Max Born famously recognised, upon seeing this draft, that the theory could be represented in terms of matrices. Soon, Born and Jordan (1925) had formulated the observables of quantum mechanics as self-adjoint or 'Hermitian' operators. ${ }^{2}$ In a letter to Jordan in September of that year, Heisenberg wrote, "Now the learned Göttingen mathematicians talk so much about Hermitian matrices, but I do not even know what a matrix is". "As Heisenberg's letter reveals, matrices were far from common tools among physicists at the time, let alone Hermitian ones, despite the latter having been introduced by Hermite (1855) seventy years earlier.

Physically significant non-Hermitian matrices appeared the following May, when London (1926) derived the non-Hermitian raising and lowering operators for the harmonic oscillator. By December of 1926, Jordan (1927a) was actually toying with the idea of treating non-Hermitian operators as observables. Remarkably, Jordan's formalism allowed one to assign complex expectation values to such nonHermitian operators, as Duncan and Janssen $(2013, \S 2.4)$ have shown. But in April of 1927, Hilbert, von Neumann and Nordheim had identified self-adjoint operators as appropriate for ensuring that the values of energy are always positive numbers. ${ }^{4}$ By

\footnotetext{
${ }^{2}$ Charmingly, their collaboration apparently began by chance, on a train to Hannover soon after Born met Heisenberg in 1925. Born recalls confiding to a colleague on the train that he had formulated Heisenberg's equations of motion using matrix theory, but was stuck trying to derive the energy from this. Jordan, who was sitting opposite and overheard the conversation, said, "Professor, I know about matrices, can I help you?" Born suggested they give it a try, and a historic collaboration ensued (from an interview with Born by Ewald 1960).

${ }^{3}$ Quoted from Jammer (1996, p.207) The impressive list of 'learned mathematicians' at Göttingen when Heisenberg arrived in 1925 includes Paul Bernays, Max Born, Richard Courant, David Hilbert, Pascual Jordan, Emmy Noether, Lothar Nordheim, B.L. Van der Waerden, and Hermann Weyl.

${ }^{4}$ (Hilbert et al. 1928). As Janssen and Duncan point out, this article was submitted in April 1927, but "for whatever reason" not published until 1928 (Duncan and Janssen 2013, §3, p.221).
} 
the time Jordan (1927b) submitted a follow-up paper in June, he had given up on the idea of non-Hermitian observables in favour of the new dogma. ${ }^{5}$

Like many aspects of quantum theory as we know it, self-adjointness was consolidated at the September 1927 Solvay conference, where Born and Heisenberg's report argued that, "the analogy with classical [Fourier] theory leads further to allowing as representatives of real quantities only matrices that are Hermitian" (Born and Heisenberg 2009, p.327). Their idea is a familiar one: it is often convenient to use a complex unit $e^{i \theta}=\cos \theta+i \sin \theta$ to represent a harmonic phenomenon like a classical wave, on the understanding that a physical wavecrest is described by just the real part, $\operatorname{Re}\left(e^{i \theta}\right)=\cos \theta$.

The dogma soon became encoded in the influential textbooks of the field, including Dirac's famous Principles of Quantum Mechanics. In the 1930 first edition, Dirac actually used the term 'observables' to refer to all linear operators. But he quickly revised this language by the second edition of 1935, writing, "it is preferable to restrict the word 'observable' to refer to real functions of dynamical variables and to introduce a corresponding restriction on the linear operators that represent observables" (Dirac 1935, p.29). The 'corresponding restriction' was that observables be self-adjoint (for a more detailed discussion of Dirac's view on observables, see Roberts 2017).

Dirac's dictum has continued to be a pervasive dogma, with an emphasis on the claim that observables have a real spectrum, as indicated in the textbook comments of the previous section. I will argue that it should be abandoned. To be precise about what I'm advocating, let me begin by setting out what I take the interpretive significance of an observable to be. I will then set out a few mathematical definitions and prerequisites that discussions of observables are not always sensitive to.

\footnotetext{
${ }^{5}$ See Duncan and Janssen $(2009,2013)$ for a fascinating exposition of this episode in the development of quantum mechanics.
} 
2.2. What we mean by 'observable'. The interpretive perspective on observables adopted in this paper will be somewhat more general than existing accounts in philosophy and physics. For example, Reichenbach $(1944, \S 6)$ proposed restricting observables to occurrences that can be directly verified using human sense organs, such as the positions of the spectral lines produced by a light source. He preferred the term 'phenomena' for occurrences that might be only indirectly observed, like the emission of a photon from a Hydrogen atom, and 'interphenomena' for everything in between. Among physicists, Reichenbach's perspective is most closely associated with the 'operational quantum physics' programme proposed by Ludwig $(1971,1983)$, and developed by Busch, Grabowski and Lahti (1995a), among others, for which observables are associated with situations for which there are probability measures over experimental outcomes.

The approach to observables in this paper will borrow a few essential elements from these ideas, but without the insistence on operationalism or on the existence of a probability measure. Our concern will rather be with Reichenbach's category of 'phenomena', and how we assign symbols to represent such phenomena. In particular, we will take an observable to minimally be a relationship between occurrences in the physical world on the one hand, and linguistic or mathematical structures used to label those occurrences. More carefully, observables will be taken to involve at least the following two components.

(1) A set of possible physical occurrences or outcomes associated any initial setup of an experiment. For example, each element of the set could represent the deflection of a particle to a location on a detection screen. By allowing the set to contain more than one element, we allow for the possibility of indeterminism in physics.

(2) A mathematical or linguistic structure that serves to label each occurrence. For example, to describe the possible locations of a particle on a spatial axis, a subset of the real line may be appropriate; similarly, a pair of spin outcomes 
may be associated with the numbers $\pm \hbar / 2$. Such symbols may have varying degrees of structure, such as ordering or algebraic relations. In general, the structure we associate with a symbol set will depend on the nature of the physical magnitudes that we intend to represent.

I do not wish to identify sufficient conditions for an observable at this stage, since the remainder of this paper will be devoted to exploring the available possibilities towards this end. However, to be clear about what we are trying to interpret, I would like to use the two components above to propose a necessary condition on observables:

An observable is (at least) an assignment of symbols in a mathematical or linguistic structure to a set of physical occurrences associated with an experimental setup.

This necessary condition on observables amounts to what philosophers often call a representation. It plays an essential role in the interpretation of quantum observables. For example, consider any of the classic models of textbook quantum theory that refer to some self-adjoint operator $A$ as an 'observable'. Such a statement satisfies our minimal criterion so long as it implicitly assumes an association between language and reality: for example, distinct experimental outcomes may be associated with distinct eigenstates of $A$, while registered values on a detector are associated with the corresponding eigenvalues. Alternatively, experimental outcomes might be associated with elements of a projection valued measure, or more generally of a positive operator valued measure (to be discussed below). In general, a great deal of modelling and physical experiment is required to establish such associations between physical occurrences and mathematical language in an interesting way. However, at this stage, our minimal requirement is only that some such association exist.

Note that the perspective here is considerably more general than Reichenbach's. His restriction to physical processes carried out and observed by a human prohibits one from describing most processes inside the sun, or in the Andromeda 
galaxy 2.5 million light years away, since neither are accessible to direct human experiment. For the sake of generality, our discussion of observables here will make no such prohibitions, although I am sympathetic to the concerns of operationalism. I have also avoided saying anything at the outset about the extent to which an observable is associated with statistics, unlike the operational approach to quantum theory (see Section 4.1). Further requirements on observables may of course be proposed. However, at this stage, I would like to adopt a definition that is general enough to allow the study of various additional conditions on what an observable may be like. These conditions are the subject of the next subsection.

2.3. Mathematical prerequisites. The mathematics of our discussion will deal entirely with Hilbert spaces over the complex field that admit a countable (though possibly infinite) basis. Some of the Hilbert space operators we discuss will be unbounded, which implies that their domains are not equal to the entire Hilbert space. When that is the case, I will still presume that they are at least densely-defined and closed. ${ }^{6}$ I will write $A^{*}$ to denote the adjoint $^{7}$ of $A$; some authors denote this with the dagger ' $\dagger$ '. An operator $A$ is called normal if and only if $A A^{*}=A^{*} A$, where we implicitly assume in this equation that $A A^{*}$ and $A^{*} A$ have the same domain. It is symmetric if it has the property that $A \psi=A^{*} \psi$ for all $\psi$ in the common domain of $A$ and $A^{*}$. It is self-adjoint if it is both symmetric and has the property that the $A$ and $A^{*}$ have the same domain. ${ }^{8}$ The term 'Hermitian' is sometimes used for one or both of these last two properties; this is unambiguous if $A$ is bounded, in which case an operator is symmetric if and only if it is self-adjoint. But since this equivalence

\footnotetext{
${ }^{6}$ An operator $A$ is densely-defined iff its domain $D_{A}$ is dense; this ensures that the operator is minimally well-defined on 'most' vector states. It is closed iff for any sequence $\left\{\phi_{n}\right\} \subseteq D_{A}$ such that $\phi_{n} \rightarrow \phi$ and $A \phi_{n} \rightarrow \psi$, it follows that $\phi \in D_{A}$ and $\psi=A \phi$. This ensures that the spectrum is allowed to be non-trivial; if a densely defined operator is not closed then its spectrum is $\operatorname{Sp}(A)=\mathbb{C}$. ${ }^{7}$ The adjoint of $A$ is defined by $A^{*} \psi:=\tilde{\psi}$, where $\langle\tilde{\psi}, \phi\rangle=\langle\psi, A \phi\rangle$ for all $\phi$ in the domain of $A$. The domain of $A^{*}$ consists of those vectors $\psi$ for which such an element $\tilde{\psi}$ exists.

${ }^{8}$ Every symmetric operator satisfies $D_{A} \subseteq D_{A^{*}}$. So, the additional condition that $D_{A}=D_{A^{*}}$ is equivalent to the statement that $D_{A^{*}} \subseteq D_{A}$. See Blank et al. $(2008, \S 4)$.
} 
fails for unbounded operators, I will try to reduce confusion by avoiding the term 'Hermitian'.

The spectrum of a linear operator $A$ is the set of numbers $\lambda \in \mathbb{C}$ such that the operator $(A-\lambda I)$ does not admit an inverse. The eigenvalues of $A$ are the subset of the spectrum consisting of elements $\lambda$ that satisfy $A \psi=\lambda \psi$ for some $\psi$. We say that an operator has a discrete or pure point spectrum when its spectrum consists entirely of eigenvalues. All operators on a finite-dimensional Hilbert space have a discrete spectrum, but in the infinite-dimensional case the spectrum may contain elements that are not eigenvalues. Finally, an important fact for our discussion is that in general, if $A$ is self-adjoint, then its spectrum (and thus its set of eigenvalues) is entirely composed of real numbers.

How do normal operators, symmetric operators, and operators with a real spectrum underpin the property of self-adjointness? One answer is given by following.

Fact. A closed, densely-defined linear operator $A$ is self-adjoint if it satisfies any two of the following properties.

(1) Normal. $A A^{*}=A^{*} A$.

(2) Symmetric. $A \psi=A^{*} \psi$ for all $\psi \in D_{A}$.

(3) Real spectrum. $\operatorname{Sp}(A) \subseteq \mathbb{R}$.

Conversely, every self-adjoint operator satisfies all three of the properties above.

This conveniently summarises several standard results. ${ }^{9}$ Self-adjoint operators are widely known to satisfy all three of the properties above. However, no single one of them is in general sufficient to guarantee that $A$ is self-adjoint: A normal operator can fail to be symmetric; a unitary operator is an example. A symmetric operator that is unbounded can fail to be normal; the so-called 'maximal symmetric' operators (operators with no self-adjoint extension) are an example. And an operator with a

\footnotetext{
${ }^{9} \mathrm{~A}$ normal operator is symmetric if and only if it is self-adjoint (Blank et al. 2008, Thm. 4.3.1); a symmetric operator has a real spectrum if and only if it is self-adjoint (Reed and Simon 1975, p.136, Thm. X.1(3)); and a normal operator has a real spectrum if and only if it is self-adjoint (this follows immediately from the spectral theorem for normal operators; see Rudin 1991, Thm. 12.26).
} 
real spectrum can fail to be symmetric. We will discuss more concrete examples of such operators over the course of this paper. But to keep the facts in one's head, it is helpful to refer to the Venn diagram of Figure 2.

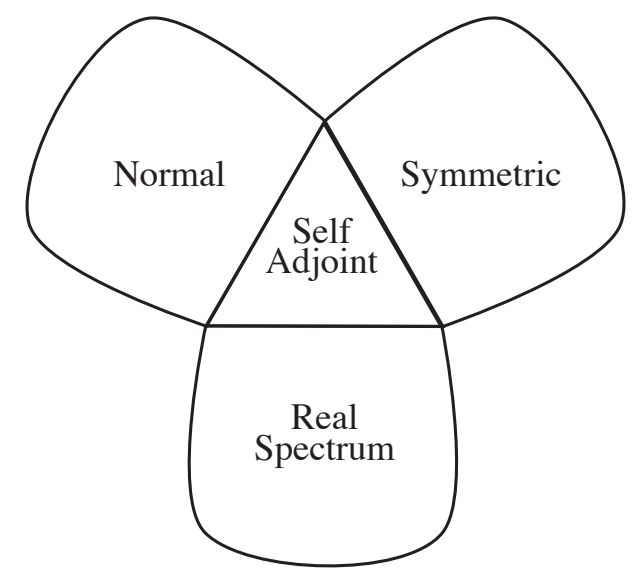

FiguRE 2. Venn diagram of normal, symmetric, and real-spectrum operators, any two of which imply self-adjointness. For bounded operators, being symmetric is equivalent to being self-adjoint, and so the right 'petal' vanishes.

Since many find the last property particularly surprising, that a non-selfadjoint operator may have a real spectrum, let me give a concrete example. A particularly simple one is the $2 \times 2$ matrix,

$$
A=\left(\begin{array}{ll}
1 & 1 \\
0 & 2
\end{array}\right) .
$$

It is obviously not symmetric (and thus not self-adjoint), since the conjugate-transpose is given by $A^{*}=\left(\begin{array}{ll}1 & 0 \\ 1 & 2\end{array}\right) \neq A$. But one can easily check that it has exactly two eigenvalues, both of which are real: $\lambda=1$ with eigenvector $\left(\begin{array}{l}1 \\ 0\end{array}\right)$, and $\lambda=2$ with eigenvector $\left(\begin{array}{l}1 \\ 1\end{array}\right)$. As expected, this operator fails to be normal, as one can verify by checking $A A^{*} \neq A^{*} A$. It also has the property that its eigenvectors span the Hilbert space, but are not orthogonal.

2.4. A classification of non-self-adjoint observables. This mathematical discussion suggests a classification scheme for non-self-adjoint observables. A consequence 
of the fact above is that all of the non-self-adjoint operators (that are closed and densely defined, as will be assumed throughout) fall into exactly one of the following four categories.

(1) Normal operators that are non-symmetric and have non-real spectra;

(2) Symmetric operators that are not normal and have non-real spectra;

(3) Real-spectrum operators that are not normal and not symmetric;

(4) None of the above: operators that fail to have all three of these properties.

That is, one can allow non-self-adjoint observables to include operators from exactly one of the three 'petals' in the flower of Figure 2, or none of them. Note that if one asserts that an observable is represented by a single operator that is bounded, then the symmetric petal vanishes, since for bounded operators being symmetric is equivalent to being self-adjoint. ${ }^{10}$

I will discuss each of these four classes of non-self-adjoint observables in turn. They introduce varying degrees of conceptual difficulties, but I will identify circumstances in which each of them are reasonable.

\section{Normal OPERATORS AS OBSERVABLES}

A simple example of a normal operator with a pure imaginary spectrum is $i Q$, where $Q$ is the position operator (for one spatial dimension) in the Schrödinger representation. It obviously commutes with its adjoint $(i Q)^{*}=-i Q$ and shares the same domain, so it is normal. Its spectrum is a line in the complex plane (namely, the pure imaginary axis) and so it can be used to represent the position of a bead in one dimension of space. It even satisfies a natural commutation relation: if we represent momentum by $i P$, then $[i Q, i P] \psi=-[Q, P] \psi=-i \psi$ (working in units of $\hbar=1$ ). Another example is the unitary operator $e^{i Q}$ : it also commutes with its adjoint, and has a spectrum equal to the complex unit circle. It can be used to represent the

\footnotetext{
${ }^{10}$ It is perhaps worth emphasising that we make no such assumption at this stage: an this account, an observable may be represented by an unbounded operator, or by a multiplicity of operators like a POVM. As we discuss in Section 4.1, each of these cases introduces observables that are symmetric but not self-adjoint.
} 
position of a bead on the loop depicted in Figure 1. And it too can be given a natural commutation relation. ${ }^{11}$

My main argument in this section is that a normal operator can be adopted as an observable in just the same sense that a self-adjoint operator can. Others have suggested this as well, ${ }^{12}$ but I will try to give a systematic argument. I begin by identifying how one can still apply the statistical rules of quantum theory to an individual normal operator, but note that a collection of normal operator observables must be restricted using the concept of a 'sharp set'. I finally discuss how symmetries and unitary evolution appear when normal operators are observables.

3.1. Eigenvectors and eigenvalues. As discussed in Section 2.2, observables capture the representation of physical facts using symbols. However, nothing about this practice requires the symbols to be real numbers; quantitative information can be conveyed by complex numbers as well, and by many other structures. For example, consider a Stern-Gerlach experiment, in which a fermion may deflect up or down as it passes through an asymmetric magnetic field. We typically label the 'deflect up' outcome with +1 and 'deflect down' with -1 , corresponding to the eigenvalues of a Pauli matrix like $\sigma_{z}:=\left({ }^{1}-1\right)$. But we could just as well label these outcomes using the pure imaginary numbers $+i$ and $-i$, which are eigenvalues of the 'anti-hermitian' matrix $i \sigma_{z}$, illustrated in Figure 3 .

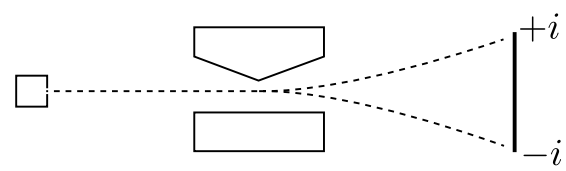

Figure 3. The outcomes of a Stern-Gerlach experiment represented by $\pm i$.

\footnotetext{
${ }^{11}$ One could simply take it to be given by the canonical commutation relations in Weyl form, $e^{i a P} e^{i b Q}=e^{i a b} e^{i b Q} e^{i a P}$. Lévy-Leblond (1976) suggests an alternative expressed in terms of angular momentum.

${ }^{12}$ See especially Lévy-Leblond (1976), Penrose (2004, p.539), and Duncan and Janssen (2013, §2.4); this latter paper shows that normal operators can be used to formalise Jordan's early theory of non-self-adjoint observables.
} 
The statistics for such an experiment can be defined just as they are in orthodox quantum mechanics: let $A$ be an operator on a Hilbert space of finite dimension, with a complex eigenvalue $\lambda$ corresponding to the eigenstate $\varphi$. Then the transition probability from an arbitrary vector state $\psi$ to $\varphi$ is still given by the usual Born rule, $|\langle\varphi, \psi\rangle|^{2}$. If the eigenvectors of $A$ form a complete basis, then its expectation value when the state $\psi$ is prepared can still be defined by $\langle\psi, A \psi\rangle=\sum_{i=1}^{n} \lambda_{i}\left|\left\langle\varphi_{i}, \psi\right\rangle\right|^{2}$, or more generally by $\operatorname{Tr}(\rho A)$ when the state is a density matrix $\rho$. For a normal operator $A$, such an expectation value may be a complex number, but this makes good conceptual sense: a complex expectation value is just a weighted average of the complex numbers representing these states.

3.2. Spectral resolution. To confirm that the practice I am proposing has the same statistical interpretation as orthodox quantum theory, we make use of the spectral theorem. This is expressed in terms of a projection valued measure (or 'spectral' measure) on Borel sets ${ }^{13}$, called the spectral resolution of the observable. In its statement for (possibly unbounded) self-adjoint operators, it says that every self-adjoint operator $A$ admits a unique projection valued measure $\Delta \mapsto E_{\Delta}$ on Borel sets of the reals such that $A=\int_{\mathbb{R}} \lambda d E_{\lambda}$, where a bounded operator $B$ commutes with $A$ if and only if $B$ commutes with each projection $E_{\Delta}$ (Blank et al. 2008, Theorem 5.3.1). In finite dimensions, the integral gets expressed as the sum,

$$
A=\sum_{i}^{n} \lambda_{i} E_{i},
$$

where each $\lambda_{i}$ is a real-number eigenvalue of $A$, and the projections $E_{i}$ satisfy $\sum_{i}^{n} E_{i}=$ 1 , and also $E_{i} E_{j}=0$ when $i \neq j$. One of the conceptually important consequences of this theorem for quantum theory is that it allows us to view each state as defining a probability distribution on definite experimental outcomes associated with $A$. For

\footnotetext{
${ }^{13} \mathrm{~A}$ projection valued measure on Borel subsets of a topological field $\mathbb{F}$ is a map $\Delta \mapsto E(\Delta)$, which associates each Borel subset $\Delta$ of $\mathbb{F}$ with a projection operator $E(\Delta)$, where $E(\mathbb{F})=I$ and $E\left(\bigcup_{i} \Delta_{i}\right)=\sum_{i} E\left(\Delta_{i}\right)$ weakly converges for any countable disjoint collection $\left\{\Delta_{1}, \Delta_{2}, \ldots\right\}$. It follows from this that $E(\varnothing)=0$, and $E\left(\Delta_{1}\right) E\left(\Delta_{2}\right)=0$ for disjoint $\Delta_{1}, \Delta_{2}$.
} 
example, in the finite-dimensional case, the spectral theorem implies there is a set of orthogonal, unit-norm eigenvectors $\varphi_{1}, \varphi_{2}, \ldots, \varphi_{n}$ of $A$ that form a basis for the Hilbert space. That fact is what allowed Born to view a vector $\psi$ as defining a probability distribution $p_{\psi}\left(\varphi_{i}\right):=\left|\left\langle\varphi_{i}, \psi\right\rangle\right|^{2}$, since it implies $\sum_{i}^{n} p_{\psi}\left(\varphi_{i}\right)=1$. Messiah thus writes in his classic textbook that,

"[a]ll... operators do not possess a complete, orthonormal set of eigenfunctions. However, the Hermitian operators capable of representing physical quantities possess such a set. For this reason we give the name 'observable' to such operators" (Messiah 1999, §V.9).

But in fact, by Messiah's reasoning, we should give the name 'observable' to normal operators, too! The more general form of the spectral theorem turns out to hold for normal operators, so that all normal operators possess a 'complete, orthonormal set of eigenfunctions' of the kind Messiah demands.

The generalisation of the spectral theorem to normal operators holds in both bounded and unbounded cases (Conway 1990, Theorem X.4.11). The proof is particularly simple in the bounded case: a bounded linear operator $A$ can always be written $A=B+i C$ with $B$ and $C$ self-adjoint, by defining $B:=\frac{A^{*}+A}{2}$ and $C:=\frac{i\left(A^{*}-A\right)}{2}$. It follows that $A A^{*}-A^{*} A=2 i(C B-B C)$, which implies that $A$ is normal if and only if $B C=C B$. The operators $B$ and $C$ can thus be viewed as simultaneously measurable, and we can derive a spectral resolution for the normal operator $A$ by applying the ordinary spectral theorem to $B$ and $C$ individually. ${ }^{14}$ In finite dimensions, this gets expressed as,

$$
A=\sum_{i}^{n} \zeta_{i} G_{i}
$$

\footnotetext{
${ }^{14}$ Let the projection-valued measures for $B$ and $C$ be $\Delta \mapsto E_{\Delta}$ and $\Delta \mapsto F_{\Delta}$, respectively. Since $B$ and $C$ commute, their projections all commute as well. This allows one to define a projection-valued measure on $\mathbb{R}^{2}$ in terms of the direct product $\Delta \times \Delta^{\prime} \mapsto G\left(\Delta \times \Delta^{\prime}\right):=E_{\Delta} \times F_{\Delta^{\prime}}$. The Borel sets of $\mathbb{R}^{2}=\mathbb{R} \times \mathbb{R}$ are naturally identified with those of $\mathbb{C}=\mathbb{R} \times i \mathbb{R}$, which finally provides the spectral resolution $A=B+i C=\int_{\mathbb{C}} \zeta d G_{\zeta}$.
} 
where each $\zeta_{i}$ is a complex-number eigenvalue of $A$, and where the properties of the projections $G_{i}$ carry over exactly as in the self-adjoint case.

This means that, just as with self-adjoint operators, every state defines a probability distribution on the experimental outcomes associated with a normal operator $A$. And just as with self-adjoint operators, a normal operator $A$ in finite dimensions has a set of orthonormal eigenvectors that form a basis for the Hilbert space, with $p_{\psi}\left(\varphi_{i}\right):=\left|\left\langle\varphi_{i}, \psi\right\rangle\right|^{2}$ defining a probability distribution over those eigenvectors.

This sort of thinking led Roger Penrose to suggested that we may relax the ordinary dogma about self-adjoint (Hermitian) observables, and adopt normal operators as well:

"In my opinion, this Hermitian requirement on an observable $Q$ is an unreasonably strong requirement, since complex numbers are frequently used in classical physics.... Since I am happy for the results of measurements (eigenvalues) to be complex numbers, while insisting on the standard requirement of orthogonality between the alternative states that can result from a measurement, I shall demand only that my quantum 'observables' be normal linear operators, rather than the stronger conventional requirement that they be Hermitian." (Penrose 2004, p.539)

Lévy-Leblond (1976) gave a similar proposal, pointing out that since a self-adjoint operator has spectral decomposition $A=\sum_{i} \lambda_{i} E_{i}$, every Borel function $f$ of a selfadjoint operator does too. A slightly different argument for normal operators originates with Dirac (1947, p.34-35), who argued that an operator in form $A=B+i C$ with $B, C$ self-adjoint is an observable only if $B$ and $C$ commute; as we have seen, this also amounts to the assertion that an observable must be represented by a normal operator $^{15}$.

\footnotetext{
${ }^{15}$ Curiously, Dirac nevertheless concludes that observables must be self-adjoint operators; the details of this episode are discussed by Roberts (2017).
} 
However, there is an important caveat to the proposal to treat normal operators as observables, to which we will now turn.

3.3. Not all normal operators: Sharp sets. The discussion above shows that any individual normal operator can be treated as an observable. However, there is also a sense in which we cannot interpret all the normal operators as observables at once, if we wish to accurately capture what is 'unobservable' in quantum theory. Let's begin with a concrete example of the problem.

Follow the proposal above: instead of using the real numbers \pm 1 to represent the $z$-spin up and down outcomes of a Stern-Gerlach experiment, let's use $\pm i$, thus adopting the 'anti-Hermitian' operator $i \sigma_{z}$ as an observable. It has the same eigenvectors as $\sigma_{z}$, but with eigenvalues $\pm i$, and is therefore not self-adjoint. This by itself amounts to little more than a relabelling of outcomes.

But the Stern-Gerlach experiments showed considerably more than this: in particular, they found it is impossible to observe spin jointly in two orthogonal directions (such as $x$ and $y$ ). This fact, now known to be one of the defining features of a quantum system, should certainly appear in our formalism. And indeed, it be reasonably captured by asserting that, if $\sigma_{x}$ and $\sigma_{y}$ are observables, then the product $\sigma_{x} \sigma_{y}$ is unobservable. This follows immediately on the orthodox perspective, since the product $\sigma_{x} \sigma_{y}$ is not self-adjoint. The problem is: the Pauli operators satisfy the relations,

$$
\sigma_{y} \sigma_{z}=i \sigma_{x} \quad \sigma_{z} \sigma_{x}=i \sigma_{y} \quad \sigma_{x} \sigma_{y}=i \sigma_{z}
$$

So, by excluding $\sigma_{x} \sigma_{y}$ from our set of observables, we also exclude $i \sigma_{z}=\sigma_{x} \sigma_{y}$.

Of course, there is no a priori requirement that the product $A B$ represent a joint measurement of $A$ and $B .{ }^{16}$ Then it simply isn't clear what $A B$ represents, in which case it fails to satisfy our minimal requirement of Section 2.2. But for present purposes, I would like to embrace the discoveries of Stern and Gerlach: certain

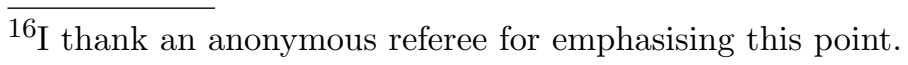


observations are impossible in quantum theory, such as the joint observation of spin in orthogonal directions, and this should be represented explicitly in our formalism. In particular, let us say that if $A$ and $B$ are non-commuting observables, then $A B$ is an unobservable. This is really just a generalisation of orthodoxy: adopting only selfadjoint observables, it follows automatically that the product of two non-commuting self-adjoints is not self-adjoint: $(A B)^{*}=B^{*} A^{*}=B A \neq A B$. However, when we open up observables to include normal operators, this separate definition is needed to represent such unobservables explicitly.

One might think that this last point is an argument for in favour of orthodoxy. But that would be too quick: the same situation can be described from the perspective of other observables as well. Suppose that we interpret the operators $\left\{i \sigma_{x}, i \sigma_{y}, \sigma_{z}\right\}$ as observables, only one of which is self-adjoint. No one of these operators is the product of the other two, and so there is no problem of the kind we have just seen. However, by taking pairwise products of each, we do find that,

$$
\left(i \sigma_{y}\right)\left(i \sigma_{x}\right)=i \sigma_{z} \quad \sigma_{z}\left(i \sigma_{y}\right)=\sigma_{x} \quad\left(i \sigma_{x}\right) \sigma_{z}=\sigma_{y}
$$

Since each of these is a product of non-commuting observables, it follows from the discussion above that each can be associated with an unobservable. Thus, although we may freely choose to interpret sets of normal operators like $\left\{\sigma_{x}, \sigma_{y}, \sigma_{z}\right\}$ and $\left\{i \sigma_{x}, i \sigma_{y}, \sigma_{z}\right\}$ as observables, we are not free to interpret both to be observables at once.

To make this restriction more systematic, let us formulate a new definition. When a set of normal operators has the special character that each element can represent an observable in the same interpretation, without introducing any unobservables, I will call it a 'sharp set'. More precisely:

Definition. A sharp set $\mathcal{S}$ of linear operators is one such that, for any $A, B \in \mathcal{S}$, if $A B \in \mathcal{S}$, then $A B=B A$. A maximal sharp set $\mathcal{S}$ with respect to a set of operators $\mathcal{A}$ is one such that $\mathcal{R} \subseteq \mathcal{A}$ is a sharp set with $\mathcal{S} \subseteq \mathcal{R}$ only if $\mathcal{R}=\mathcal{S}$. 
A more careful proposal for treating normal operators as observables is then to say: a set of normal operators consists of observables only if it is a sharp set. This set is 'as big as it can be' if only if it is maximal. We may immediately identify a few easy implications of this proposal.

Proposition 1. The following properties hold of sharp sets on a Hilbert space $\mathcal{H}$.

(1) A set that is closed under multiplication is sharp iff it is commutative.

(2) The set of all normal operators is not in general a sharp set.

(3) Every set of self-adjoint operators is a sharp set.

(4) The sharp set of self-adjoint operators in $\mathcal{B}(\mathcal{H})$ is not maximal in $\mathcal{B}(\mathcal{H})$.

Proof. (1) is immediate from our definition, and (2) follows from the discussion above. (3) If $\mathcal{S}$ is a set of self-adjoint operators and $B, C \in \mathcal{S}$, then $B C \in \mathcal{S}$ only if $B C=$ $(B C)^{*}=C B$, which means that $\mathcal{S}$ is sharp. (4) For any set $\mathcal{S}$ of self-adjoint operators, let $\mathcal{R}=\mathcal{S} \cup\{i I\}$ with $I$ the identity operator. Let $A, B, A B \in \mathcal{R}$. If either $A=i I$ or $B=i I$, then clearly $A B=B A$. It thus remains to show that $A B=B A$ when $A, B \in \mathcal{S}$. But no pair of self-adjoint operators $A, B \in \mathcal{S}$ can satisfy $A B=i I$, since $A B$ and $(A B)^{*}=B A$ have the same non-zero spectral elements, whereas $i I$ and $(i I)^{*}$ do not. In this case we thus have $A, B, A B \in \mathcal{S}$, which implies that $A B=B A$ by the previous argument.

This last property suggests in particular that, by associating observables with the concept of a maximal sharp set in place of the standard set of self-adjoint operators, it is possible to meaningfully enrich the standard observables beyond what is usually available. In this sense, the self-adjoint operators are not as 'large as they could be'. Not all normal operators can be treated as observables at once, but we can certainly include more than just the self-adjoint ones. On the other hand, whether or not there is a non-trivial sharp set that extends the self-adjoint operators remains an open question. 
I have argued the 'normal operator' approach to observables advocated by Penrose and others is improved by a restriction to sharp sets. However, the appropriate reaction to sharp sets remains up for debate. One may decide that the complexity of the whole matter indicates that normal operators are more trouble than they're worth. ${ }^{17}$ Alternatively, one may conclude that the transformations between sharp sets introduce an interesting new symmetry into quantum theory, or that the set of all observables must include more than just self-adjoint operators. Although I am optimistic about this second perspective, its success may depend on further exploration of the structure of sharp sets in quantum theory. For the moment, much remains to be learned about that structure.

3.4. Symmetries and Dynamics. A final question about normal operators as observables is how one ought to understand symmetries in this context. In orthodox quantum theory, there is a tight connection between symmetries and self-adjoint operators, which is reminiscent of Noether's theorem for variational symmetries. Namely, Stone's theorem guarantees a continuous group of symmetries is always generated by a unique self-adjoint operator. More precisely, if $s \mapsto U_{s}$ is a strongly continuous, one-parameter set of unitary operators satisfying $U_{r} U_{s}=U_{r+s}$ for all $r, s \in \mathbb{R}$, then there exists a unique self-adjoint operator $A$ such that $U_{s}=e^{i s A}$ for all $s \in \mathbb{R}$ (Blank et al. 2008, Thm. 5.9.2). Conversely, every self-adjoint operator generates a strongly continuous one parameter unitary representation of this kind. Examples: the spatial translation group $s \mapsto U_{s}$ is generated by the momentum operator $P$, in that $U_{s}=e^{i s P}$. Similarly, the spatial rotation group $\theta \mapsto R_{\theta}$ is generated by the angular momentum operator $J$, in that $R_{\theta}=e^{i \theta J}$.

Can Stone's theorem be converted into an argument that observables must be self-adjoint operators? One might try to argue that continuous symmetries are generally associated with a conserved quantity, which we should think of as an observable.

\footnotetext{
${ }^{17}$ Thanks to [BLINDED FOR REVIEW] and an anonymous referee for this point.
} 
This does allow one to identify certain self-adjoint operators as observables. For example, the expectation value of momentum $P$ does not change under spatial translations, since the fact that $P$ and $U_{s}$ commute implies that for any (pure or mixed) state represented by a density operator $\rho$, we have $\operatorname{Tr}\left(U_{s} P U_{s}^{*} \rho\right)=\operatorname{Tr}\left(U_{s} U_{s}^{*} P \rho\right)=\operatorname{Tr}(P \rho)$. However, this thinking works for normal operators, too: a whole host of normal operators are conserved along continuous unitary symmetries. Indeed, if $U_{s}$ is generated by the self-adjoint operator $A$, then every Borel function $f(A)$ is also similarly conserved, since such a function $f(A)$ always commutes with $e^{i s A}$. As a result, nonself-adjoint normal operators like $i P$ and $e^{i a P}$ are conserved along spatial translations just as much as the momentum operator $P$ is. So, conservation is no argument that observables must always be self-adjoint operators. And after all, strictly speaking, the generator of a unitary group $U_{s}=e^{i s A}$ is not really a self-adjoint operator, but rather the 'pure imaginary' operator $i A$, which has only imaginary numbers in its spectrum.

Still: even if there are non-self-adjoint normal observables, one might insist on an ordinary unitary dynamics, which requires a self-adjoint generator $H$ (the 'Hamiltonian'). The reasoning can be made precise as follows. We might begin by assuming that, like most representations of change over time, our dynamics $t \mapsto U_{t}$ is strongly continuous. Moreover, isolated systems seem to allow the same experiment to be repeated at later moments in time with the same probabilistic outcomes, which is to say that the dynamics seems to satisfy time-translation invariance as well, $U_{t_{1}+t_{2}}=$ $U_{t_{1}} U_{t_{2}}$, with $U_{t}$ unitary so as to preserve probabilities. ${ }^{18}$ Finally, suppose we presume that dynamical evolution holds (or could in principle hold) infinitely to the future and to the past, i.e. it can be described for all $t_{1}, t_{2} \in \mathbb{R}$. If one believes these things about the evolution of a quantum system, then Stone's theorem guarantees that the generator — the Hamiltonian $H$ - is self-adjoint.

\footnotetext{
${ }^{18}$ That $U_{t}$ is unitary or antiunitary for each $t \in \mathbb{R}$ follows from Wigner's theorem (Bargmann 1954; Uhlhorn 1963); that it is unitary in particular follows from the fact $U_{t}$ can always be written $U_{t}=\left(U_{t / 2}\right)^{2}$, since the square of a unitary (or an antiunitary) is always unitary.
} 
This perspective is certainly compatible with non-self-adjoint observables that are not the Hamiltonian. However, there may also be physical circumstances in which one or more of these assumptions fails. This can lead to the failure of unitarity and a failure of the Hamiltonian to be self-adjoint. For example, a non-isolated system does not satisfy the requirement of time translation invariance; we will see this example again in the discussion of radioactive decay in Section 4.3. The dynamics of such systems are not generally generated by a single self-adjoint Hamiltonian.

It may also be unreasonable to assume that dynamical evolution holds forever to the future and to the past. Such an assumption is much stronger than what is normally required of classical Hamiltonian mechanics, where only local time evolution is guaranteed. ${ }^{19}$ One might similarly expect that for some quantum systems, time translation might be defined only locally, perhaps because the system has a finite past, a finite future, or for some other reason altogether. This dynamical evolution will be generated by a Hamiltonian that is not-self-adjoint. Indeed, we will see explicit examples of this kind of evolution among the non-normal operators of the next section.

\section{NON-NORMAL OPERATORS AS OBSERVABLES}

Let us now turn to another class of non-self-adjoint observable, which involves operators that are not even normal. Treating non-normal operators as observables is a more dramatic extension of quantum theory, far from a mere adjustment of convention. Following the mathematical discussion of Section 2.4, there are three kinds of non-self-adjoint operators in this class: those that are symmetric but do not have a real spectrum; those that have a real spectrum but are not symmetric; and those that satisfy neither condition. One may therefore choose exactly one of these conditions, or else reject them both. There are circumstances in which each is reasonable, which I will discuss in turn.

\footnotetext{
${ }^{19}$ More formally: A smooth function $h: M \rightarrow \mathbb{R}$ on a symplectic manifold generates a Hamiltonian vector field, for which one can find a unique set of integral curves in a neighbourhood of each point. But it is perfectly possible for this Hamiltonian vector field to be incomplete, which is to say that its set of integral curves $\varphi(t)$ cannot be defined for all parameter times $t \in \mathbb{R}$.
} 
4.1. Symmetric operators and POVMs. Let me begin by recalling a case in which one commonly treats a non-self-adjoint operator like an observable, but only because it can be extended to a self-adjoint operator. I will then turn to the case that is more important for my purposes: non-self-adjoint operators that cannot be extended in this way.

Case 1: Self-adjoint extensions. Suppose we wish to describe a particle in a box of finite width $b-a$ in some spatial dimension. We adopt the Hilbert space $\psi \in L^{2}([a, b])$ with $\langle\psi, \phi\rangle:=\int_{a}^{b} \psi^{*}(x) \phi(x) d x$. As experienced students of quantum mechanics, we wish to see a momentum observable for this particle that looks like the standard momentum operator $P=i d / d x$. Such an unbounded operator cannot act on the entire Hilbert space. The art of unbounded operators is thus to answer the question: which wavefunctions does the operator it act on? Since the operator will have different properties depending on the domain it is defined on, let me for the moment describe momentum as an operator-domain pair $(P, D)$. Suppose we identify the domain $D$ as the subset of $L^{2}([a, b])$ consisting of at least once-differentiable functions that vanish at the edges of the box, $\psi(a)=\psi(b)=0$, as shown in Figure 4. Let $P=i d / d x$, and call this domain $D_{0}$. Then $\left(P, D_{0}\right)$ can be shown to be closed, densely-defined and symmetric; however, it is not self-adjoint (Blank et al. 2008, Example 4.2.5). It is also not normal and fails to have a purely real spectrum, as a consequence of our mathematical discussion above.

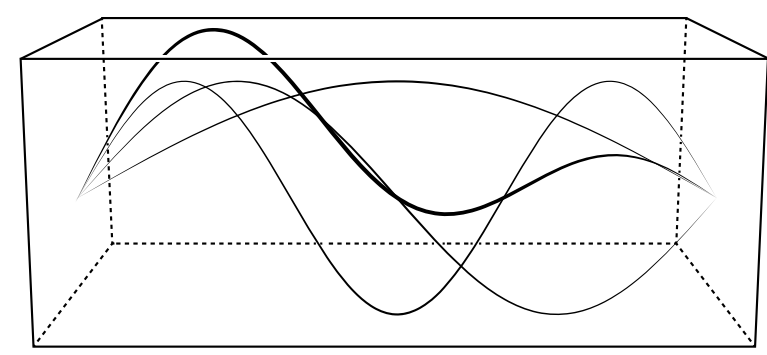

Figure 4. $P=i \frac{d}{d x}$ is not self-adjoint on differentiable wavefunctions that vanish at the sides of a box, $\psi(a)=\psi(b)=0$. 
Nevertheless, it is common practice to view non-self-adjoint operators like this one as observables. This is because one can turn $\left(P, D_{0}\right)$ into a self-adjoint operator by extending its domain, this time to include all the wavefunctions that satisfy $\psi(a)=e^{i \theta} \psi(b)$ for some fixed real $\theta$, which includes those that vanish at the edges of the box as a subset. Call this extended domain $D_{\theta} \supset D_{0}$. Then for each real number $\theta$, the pair $\left(P, D_{\theta}\right)$ is self-adjoint $(i b i d)$. This procedure is common practice: construct a symmetric observable that is physically motivated but not self-adjoint, with the aim of extending to a self-adjoint operator as needed.

Earman has pointed out that this practice can lead to a "new form of quantum indeterminism (distinct from state vector collapse)" when the observable is interpreted as generating the dynamics (Earman 2009, p.28). As in the case of the particle in a box, a symmetric operator may admit multiple distinct self-adjoint extensions. Since each such self-adjoint extension $H$ generates a distinct unitary group $U_{a}:=e^{-i t H}$, a description of this kind fails to determine the future (or past) on the basis of initial conditions.

This failure of determinism might lead one to be sceptical of treating such operators as observables. Often a symmetric operator does admit a unique self-adjoint extension, in which case it is called essentially self-adjoint. To avoid indeterminism, one might then postulate that only essentially self-adjoint operators are good candidate observables. This is not so convincing if one takes the question of determinism to be an open one, which should be addressed by physics rather than by fiat. ${ }^{20}$ But another class of non-self-adjoint symmetric operators is even more convincing. That class is the following.

Case 2: Maximal symmetric operators and POVMs. Let me turn to cases that may appear 'worse', but are in fact better: symmetric operators that do not admit any self-adjoint extensions at all. Such operators are called maximal symmetric. If

\footnotetext{
${ }^{20}$ Earman (2009, p.36) still supports the practice of treating some symmetric operators that are not self-adjoint as observables, calling its rejection "high handedness"; Wüthrich (2011, p.373) agrees for this reason that "the question of whether the Schrödinger evolution is deterministic does not afford a simple and unqualified answer."
} 
we wish to treat a maximal symmetric operator like an observable, then it cannot stand proxy for a self-adjoint extension; it must be treated like an observable in its own right.

The assumptions of Stone's theorem fail for maximal symmetric operators, so they do not generate a unitary group in the usual sense. However, they do satisfy a closely related result. Stating this result uses the concept of an isometry, that is, a Hilbert space operator $U$ for which $U^{*} U=E$ is a projection operator (a unitary operator is thus a particular isometry for which $U^{*} U=U U^{*}=I$ ). An isometry is a symmetry transformation in much the same sense as a unitary operator, but in a more restricted domain, in that $|\langle U \psi, U \phi\rangle|=|\langle\psi, \phi\rangle|$ for all $\psi, \phi \in E \mathcal{H}$. Isometries also allow one to state the following generalisation of Stone's theorem. ${ }^{21}$

Generalised Stone Theorem. If $s \mapsto U_{s}$ is a strongly continuous, one-parameter set of isometries satisfying $U_{r} U_{s}=U_{r+s}$ for all $r, s \geq 0$ (or for all $r, s \leq 0$, but not both), then there exists a unique maximal symmetric operator $A$ such that $U_{s}=e^{i s A}$. Conversely, every maximal symmetric operator A generates a strongly continuous one parameter set of isometries set $s \mapsto U_{s}=e^{i s A}$ satisfying $U_{r} U_{s}=U_{r+s}$, for all $r, s \geq 0$ (or for all $r, s \leq 0$, but not both). (Cooper 1947, 1948)

This means that maximal symmetric operators are associated with a set of symmetries after all, in much the same way as self-adjoint operators. These symmetries are simply limited to a restricted subspace, in addition to being limited by the parameter values of the set.

When a maximal symmetric observable is a Hamiltonian, the Generalised Stone Theorem says that a unique solution to the Schrödinger equation exists, although it is only defined for non-negative times or non-positive times (but not both). As far as determinism is concerned, this situation is an improvement on the failure of essential self-adjointness considered by Earman (2009). The generalised Stone

\footnotetext{
${ }^{21}$ This result follows naturally from the work of Naimark $(1940,1968)$ on the theory of self-adjoint extensions, although it was proved independently by Cooper $(1947,1948)$. The same technique turns out to allow for a notion of 'weak localizability' for relativistic photons (Jauch and Piron 1976).
} 
theorem says that the dynamical evolution generated by a maximal symmetric Hamiltonian is unique, much like the dynamics of an essentially self-adjoint Hamiltonian. The dynamics is time-translation invariant, in the restricted sense of an isometry. The limitation is merely that this dynamics is not defined for all times $t \in \mathbb{R}$. But as discussed above, having a dynamics for all times is a very strong requirement, which we may have good reason to relax.

Maximal symmetric operators also fail to satisfy the conditions of the ordinary spectral theorem. But there is an interesting generalisation of this too, which makes use of Positive Operator-Valued Measures (POVMs) ${ }^{22}$. A POVM generalises our earlier notion of a projection-valued measure, by carrying over its properties to positive operators, and in particular those self-adjoint operators with spectrum in the interval $[0,1]$. Such measures allow us to state the following.

Naimark Spectral Theorem. Let $A$ be a closed, densely defined symmetric operator. Then there exists a POVM $\Delta \mapsto F_{\Delta}$ such that $A=\int_{\mathbb{R}} \lambda d F_{\lambda}$, which is unique (up to unitary equivalence) if and only if $A$ is maximal symmetric, and which is a Projection Valued Measure if and only if $A$ is self-adjoint. (Dubin and Hennings 1990, Thm. 5.16, pg.135)

Thus, just like self-adjoint operators, maximal symmetric operators have a unique spectral decomposition. It is just not in terms of a projection valued measure, but rather a POVM. Conversely, every POVM $\Delta \mapsto F_{\Delta}$ gives rise to a symmetric operator of the form $A:=\int_{\mathbb{R}} \lambda d F_{\lambda}$. . And, just as with projection-valued measures, we can more generally define the analogue of 'normal' operators by considering, for any Borel function $f: \mathbb{R} \rightarrow \mathbb{C}$, an operator of the form $f(A):=\int_{\mathbb{R}} f(\lambda) d F_{\lambda}$.

Although we have arrived at POVMs by beginning with maximal symmetric operators, they can also be treated as a basis for understanding observables in their

${ }^{22}$ Let $\mathcal{E}(\mathcal{H})$ be a set of effects or positive (self-adjoint) operators $A$ on $\mathcal{H}$ with spectrum in the interval $[0,1]$. A Positive Operator-Valued Measure (POVM) for $\mathcal{E}(\mathcal{H})$ is a triple $(X, S, E)$, where $X$ is a set, $S$ is a $\sigma$-algebra of subsets of $X$, and $E: S \rightarrow \mathcal{E}(\mathcal{H})$ is a function that satisfies: $(1) E(\Delta)$ is positive for each $\Delta \in S ;(2) E\left(\cup_{i} \Delta_{i}\right)=\sum_{i} E\left(\Delta_{i}\right)$ for all disjoint sequences $\Delta_{i}$; and $(3) E(X)=1$. 
own right, following the proposals of Ludwig (1983) and of Busch et al. (1995a), among others. Suppose that we represent a set of experimental outcomes using a set of positive operators on a Hilbert space, for example by associating each Borel set $\Delta \subset \mathbb{R}$ with a positive operator $F_{\Delta}$. This is an observable in the general sense described in Section 2.2, and has been interpreted in this way at least since the work of Jauch and Piron (1976). One particularly elegant motivation for this perspective is the following. Given a set $X$ and a $\sigma$-algebra $S$ of its subsets, let $\mu_{\psi}: S \rightarrow \mathbb{R}$ be defined for each $\psi$ in a Hilbert space $\mathcal{H}$ by,

$$
\mu_{\psi}(\Delta):=\left\langle\psi, F_{\Delta} \psi\right\rangle
$$

It is straightforward to verify that the function $\mu_{\psi}$ is a (real-valued) measure for every $\psi \in \mathcal{H}$ if and only if the function $\Delta \mapsto F_{\Delta}$ is a POVM (Berberian 1966). ${ }^{23}$ This provides one interesting way to motivate a POVM: it is necessary and sufficient for viewing the Born rule of ordinary quantum theory as a measure associated with positive operators.

Further support for the POVM approach comes from a neat variation of Gleason's theorem. A positive operator $A$ on a Hilbert space is called an effect whenever its spectrum is in the interval $[0,1]$. This is a considerable extension of the lattice of projections. Now, Gleason's theorem shows that the probability measures on projections are completely characterised by the Born rule, so long as $\operatorname{dim} \mathcal{H} \geq 3$. But by expanding attention from projections to the larger space of effects $\mathcal{E}(\mathcal{H})$ on a Hilbert space, Busch (2003) found a similar (and much simpler) result that holds for Hilbert spaces of arbitrary dimension.

Generalised Gleason Theorem (Busch). Given a generalised probability measure ${ }^{24}$ $p: \mathcal{E}(\mathcal{H}) \rightarrow[0,1]$, there exists a density operator $\rho$ on $\mathcal{H}$ such that $p(E)=\operatorname{Tr}(\rho E)$ for all $E \in \mathcal{E}(\mathcal{H})$.

\footnotetext{
${ }^{23}$ I thank an anonymous referee for pointing this out.

${ }^{24} \mathrm{~A}$ generalised probability measure on a set of effects $\mathcal{E}(\mathcal{H})$ is a function $p: \mathcal{E}(\mathcal{H}) \rightarrow[0,1]$ such that $p(I)=1$ and $p\left(\sum_{i} E_{i}\right) \leq \sum_{i} p\left(E_{i}\right)$ whenever the countable sequence $E_{i}$ satisfies $\sum_{i} E_{i} \leq I$.
} 
Busch's theorem shows that probabilities on effects are completely characterised by the Born rule, in a manner very similar to Gleason's theorem. This provides yet more evidence that effects and POVMs thus provide an appropriate and general way to describe probabilistic experiments in quantum theory. ${ }^{25}$

POVMs also have a number of practical applications, including a more accurate description of Stern and Gerlach's famous experiment (Busch et al. 1995a, pp.7-9). However, the interpretation of an arbitrary POVM can be subtle. A curiosity about POVMs is that two elements $E(\Delta)$ and $E\left(\Delta^{\prime}\right)$ with $\Delta \cap \Delta^{\prime}=\varnothing$ are not necessarily orthogonal, or even commutative. For this reason, a POVM is sometimes interpreted as describing measurements in which the experimental outcomes may be 'fuzzy', 'unsharp' or 'overlapping', in that they are not mutually exclusive.

In spite of these interpretive subtleties, the great advantage of symmetric operators, POVMs, and effects is that they allow for the description of novel physical phenomena, which are not otherwise describable using orthodox observables. I will just mention one such description to illustrate, which is the case of 'time observables' (more examples can be found in Busch et al. 1995a).

A time observable is a natural object of study when one is interested in durations of some process, or in the time at which something occurs. For example, one might wish to use an observable to describe the moment that a particle in flight arrives at its target, or the time that a jet or particle decay occurs in a detector. However, their interpretation demands some care: suppose that two intervals of time $\Delta$ and $\Delta^{\prime}$ do not overlap, in that $\Delta \cap \Delta^{\prime}=\varnothing$, and that $F_{\Delta}$ and $F_{\Delta^{\prime}}$ are operators that (respectively) represent an occurrence in each of those intervals. Then one would not in general expect $F_{\Delta}$ and $F_{\Delta^{\prime}}$ to be orthogonal. On the contrary, an occurrence in $\Delta$ is generally compatible with an occurrence in $\Delta^{\prime}$, since the two happen at different times. Thus, time observables are a natural candidate for description in terms of a $\mathrm{POVM} \Delta \mapsto F_{\Delta}$.

\footnotetext{
${ }^{25}$ For a detailed discussion of Gleason's theorem, Busch's variation, and their proof and philosophical implications, see Landsman $(2017, \S 2)$.
} 
There is a literature that has come to this same conclusion through another route. Let $\mathcal{H}$ be a Hilbert space, together with an ordinary unitary dynamics defined by $t \mapsto U_{t}$. We call a linear operator $T$ a time operator if and only if it satisfies $U_{t} T U_{t}^{*}=T+t I$ for all $t \in \mathbb{R}$. Equivalently, for any $\psi \in D_{T}$ with $|\psi|=1$ and $\psi(t):=U_{t} \psi$, a time operator $T$ is one that satisfies $\langle\psi(t), T \psi(t)\rangle=\langle\psi, T \psi\rangle+t$, for all $t \in \mathbb{R}$. These properties can be informally summarised as requiring that a time observable 'tracks' the passing of time in the same way as the unitary dynamics. An operator $T$ with this property is in general unbounded, and also satisfies the timeenergy commutation relation $[H, T]=i$, which is the 'local' expression of $U_{t} T U_{t}^{*}=$ $T+t I$.

The central no-go result for time operators, known as Pauli's theorem, is that if $U_{t}=e^{-i t H}$ is generated by a Hamiltonian $H$ that is bounded from below (as almost all known Hamiltonians are), then every time operator fails to be self-adjoint. ${ }^{26}$ This fact was originally interpreted as an impossibility result for time observables, and is sometimes referred to as the 'problem of time' in quantum mechanics (Butterfield 2013). However, if we relax our requirements on what counts as an observable, then it can equally be viewed as simply saying that time operators are non-self-adjoint observables. Then there turn out to be a plethora of possible time observables, most known examples of which are maximal symmetric.

One simple time observable ${ }^{27}$ is well known for the Schrödinger representation on $L^{2}(\mathbb{R})$, with the dynamics defined by the free Hamiltonian $H=\frac{1}{2 m} P^{2}$. This dynamical system admits a time operator given by,

$$
T=\frac{m}{2}\left(Q P^{-1}+P^{-1} Q\right)
$$

where $P^{-1}$ is defined using the functional calculus and is self-adjoint on an appropriate domain. This $T$ is a time operator because the free particle satisfies $e^{-i t H} Q e^{i t H}=$

\footnotetext{
${ }^{26}$ This result is inspired by a famous remark of Pauli (1980, pg.63, fn.2), which was made more rigorous e.g. by Ludwig (1983, §VII.6) and Srinivas and Vijayalakshmi (1981), among others.

${ }^{27}$ This example was identified by Aharonov and Bohm (1961). For further discussion, see also Holevo (1982); Busch et al. (1994); Galapon (2009); Pashby (2014).
} 
$Q+\frac{t}{m} P$ and $e^{-i t H} P e^{i t H}=P$, from which it easily follows that $e^{-i t H} T e^{i t H}=T+t I$. It is also symmetric by construction.

However, we can immediately infer from Pauli's theorem that this time operator is not self-adjoint; and with a little more work we can show that it does not have any self-adjoint extensions (Holevo 1982, §3.8). It follows that $T$ is maximal symmetric. A large class of dynamical systems with such time operators has been constructed by Busch et al. (1994) and by Hegerfeldt and Muga (2010), and these observables have been put to many interesting uses (Muga et al. 2008). A closely-related discussion also exists for 'phase observables' (Busch et al. 1995b).

Let me summarise the discussion of this section. The addition of maximal symmetric operators or POVMs as observables is a non-trivial extension of quantum theory. However, it is a mathematically controlled extension, thanks to a collection of generalisations of Stone's theorem, the spectral theorem, and Gleason's theorem. These generalisations introduce features that are unfamiliar from the perspective of more traditional observables. However, even these unfamiliar features can be made sense of in concrete physical descriptions in which we can put maximal symmetric operators to use. Little reason remains to deny their status as bona fide 'observables'.

4.2. Real spectrum operators and PT symmetry. Among the most commonly demanded requirements on a quantum observable is that it should have a real spectrum. Non-self-adjoint operators with a real spectrum, which from the discussion of Section 2.3 we know are neither normal nor symmetric, thus provide another natural route to extending observables in quantum mechanics. However, as we shall see, this class of operators is much more unwieldy than the previous ones, with no analogue of the spectral theorem nor of Stone's theorem without adding extra structure to the theory.

We have discussed the matrix $A=\left(\begin{array}{ll}1 & 1 \\ 0 & 2\end{array}\right)$ as an example of a non-self-adjoint operator with a real spectrum. A much more interesting example from the perspective 
of physical applications is the operator,

$$
H=\frac{1}{2 m} P^{2}+\frac{m \omega^{2}}{2} Q^{2}+i Q^{3},
$$

where $Q$ and $P$ are the position and momentum operators in some representation of the canonical commutation relations, and $m, \omega \in \mathbb{R}^{+}$. This operator is obviously not symmetric, and therefore fails to be self-adjoint or even normal. However, it has been studied extensively following the work of Bender and Boettcher (1998) as a possible interaction Hamiltonian, and was proven by Dorey et al. (2001a,b) to have an entirely real spectrum (in spite of the $i Q^{3}$ term!), with interesting connections to supersymmetry.

This research is part of a more general programme known as $\mathcal{P} \mathcal{T}$-symmetric quantum mechanics, which aims to construct non-self-adjoint energy observables with an entirely real spectrum (Bender et al. 2002, 2003). Restricting to a real spectrum is plausible in this context, since energy is thought to be a linearly ordered quantity that is bounded from below. To aid with the construction of these operators, a symmetry principle is used. One generally adopts the Schrödinger representation on $L^{2}(\mathbb{R})$, defining the parity or 'mirror symmetry' operator $\mathcal{P}$ by $\mathcal{P}:=e^{(i \pi / 2)\left(Q^{2}+P^{2}\right)}$ and the time reversal operator $\mathcal{T}$ by $\mathcal{T} \psi:=\psi^{*}$ for all $\psi \in L^{2}(\mathbb{R})$. As expected, these operators satisfy $\mathcal{P} Q \mathcal{P}^{-1}=-Q=-\mathcal{T} Q \mathcal{T}^{-1}$ and $\mathcal{P} P \mathcal{P}^{-1}=\mathcal{T} P \mathcal{T}^{-1}=-P$. It turns out that, if the eigenvectors of a discrete-spectrum operator $H$ are preserved by the combined transformation $\mathcal{P} \mathcal{T}$, and if this $H$ is also invariant under $\mathcal{P} \mathcal{T}$ in that,

$$
[\mathcal{P} \mathcal{T}, H]=0
$$

then $H$ is guaranteed to have a real spectrum (Weigert 2003). However, although the criterion of $\mathcal{P} \mathcal{T}$-symmetry is sometimes presented as a requirement on physically reasonable energy observables, it is not required in order to have an observable with a real spectrum (Lévai and Znojil 2001).

Streater (2007) has discussed another general class of non-self-adjoint operators that have a real spectrum. Call a linear operator $A$ a diagon if and only if there 
exists an operator $B$ with densely-defined inverse such that $B A B^{-1}$ is self-adjoint. Since the similarity transformation $(\cdot) \mapsto B^{-1}(\cdot) B$ is spectrum-preserving, it follows that every diagon has a real spectrum. In particular, a self-adjoint operator is a diagon with $B=I$, i.e. the identity. However, diagons are certainly not always self-adjoint. For example, if $Q$ and $P$ are the position and momentum operators in the Schrödinger representation, then $Q^{-1} P Q$ is a non-self-adjoint diagon. It can be transformed to the self-adjoint operator $P$ by the similarity transformation $(\cdot) \mapsto Q(\cdot) Q^{-1}$, and thus has a real spectrum. But it is easy to check that it is neither symmetric nor normal: therefore it is not self-adjoint. ${ }^{28}$

One strange feature of non-self-adjoint diagons is that their expectation values may not be real, even though the spectrum of the operator is. In the example above, this is easy to check: for an arbitrary vector $\psi$ in the common domain of $Q, P$ and $Q^{-1}$, we have by application of the commutation relations that,

$$
\left\langle\psi,\left(Q P Q^{-1}\right) \psi\right\rangle=\left\langle\psi,\left(i Q^{-1}+P\right) \psi\right\rangle=i\left\langle\psi, Q^{-1} \psi\right\rangle+\langle\psi, P \psi\rangle .
$$

This implies that $Q P Q^{-1}$ has expectation values with a pure-imaginary component. Streater $(2007, \S 12.5)$ has pointed out that such complex expectation values are quite general features of non-self-adjoint diagons. Thus, even though these operators retain what many have taken to be the 'gold standard' of observables, a real spectrum, their expectation values may not satisfy this standard, which is difficult to interpret.

A non-self-adjoint operator with a real spectrum is never 'diagonalizable' in the usual sense: it does not have a spectral decomposition in the sense of a projection valued measure, since the ordinary spectral theorem applies only to normal operators. It is not known whether a more general spectral theorem exists for such operators, analogous to the Naimark spectral theorem for symmetric operators. The application of Stone's theorem suffers from similar difficulties.

\footnotetext{
${ }^{28}$ Apply the commutation relations to see that it is not symmetric: $\left(Q^{-1} P Q\right)^{*}=Q P Q^{-1}=i Q^{-1}+$ $P$, whereas $Q^{-1} P Q=i Q^{-1}-P$. One can use these facts to check that $Q^{-1} P Q$ is also not normal.
} 
However, the spectral structure of a large class of real-spectrum operators has been studied using other kinds of decompositions, introduced by Bender et al. (2000) and developed by Weigert (2003) and others. The usual statistical interpretation of quantum theory is not possible for these operators, since they do not admit a projection-valued measure. However, an interpretation is still possible if one introduces a new inner product, and then defines the statistics and the dynamics with respect to that. For example, if $H$ is a non-self-adjoint diagon on a Hilbert space with inner product $\langle\cdot, \cdot\rangle$, then one can always construct a new inner product $\langle\cdot, \cdot\rangle_{H}$ with respect to which $H$ is self-adjoint. One can then take spectral decompositions and define a unitary dynamics in the new resulting Hilbert space. This strategy, proposed by Bender et al. (2002), has been the subject of a great deal of fruitful research. ${ }^{29}$

We thus have an interesting extension of quantum theory, in which we only require the spectrum of each observable to be real, and each non-self-adjoint observable requires its own inner product. However, a central requirement of this programme, that one must insist on having a real spectrum, is perhaps not as well-motivated as it is sometimes made out to be. Although it is often a calculational convenience to represent linearly-ordered magnitudes using real numbers, it is also to have complex linear orderings, as well as physical magnitudes (like position on a plane) that do not exhibit a natural linear ordering at all. The previous sections have reviewed many scenarios in which non-real numbers can be used to represent physical experiments. If one is willing to relax the requirement of self-adjointness at all, then one should minimally allow for complex eigenvalues, too. This is the topic of the last section.

4.3. None of the above: The wilderness beyond. For each of the properties of being normal, being symmetric, and having a real spectrum, there is a literature on retaining that property while giving up other two. In this section, we discuss the possibility of giving up all three. Then there is no single mathematical idea controlling the concept of an observable, which leads of course to losing the usual mathematical

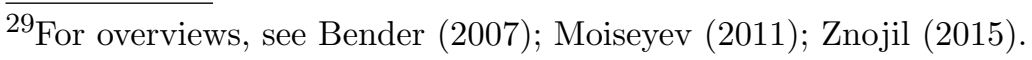


results that more traditional observables enjoy. However, the result is not necessarily a complete free-for-all. There are physical ideas that allow even these operators to be interpreted as observables, and mathematical results that allow us to control their behaviour.

An early example of such an observable was proposed by Gamow (1928), in a famous paper written on a visit to Göttingen that introduced the world to quantum tunnelling. Adopting Schrödinger's wavefunction formalism, Gamow proposed that the energy value of a radioactive particle could be described by a complex number,

$$
E=E_{0}+i \Gamma
$$

He gave an immediate physical interpretation of this value, identifying $E_{0} \in \mathbb{R}^{+}$the 'ordinary energy' and $\Gamma=\hbar \lambda / 2$ with $\lambda$ describing a 'damping term'. One then finds that a corresponding energy eigenstate $\phi$ would evolve according to the rule,

$$
\phi(t)=e^{-i t E} \phi=e^{-i t\left(E_{0}+i \Gamma\right)} \phi=e^{t \Gamma} e^{-i t E_{0}} \phi .
$$

This state is nearly stationary $\phi(t) \approx e^{-i t E_{0}} \phi$ when $t \approx 0$. Gamow takes this to indicate a damping effect on the wave amplitude soon after the wave is emitted from the atom, which then increases exponentially with time as the wave gets farther away, illustrated in Figure 5.
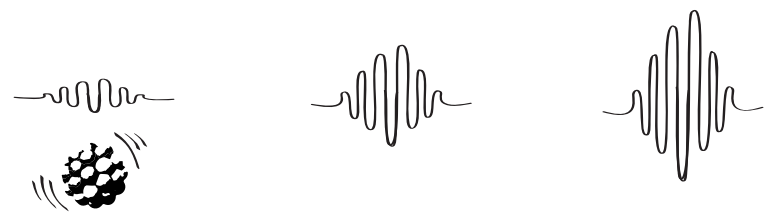

Figure 5. Gamow's (1928) model of quantum tunnelling used a nonself-adjoint Hamiltonian with eigenvalue $E=E_{0}+i \Gamma$.

What sort of observable generates the dynamics $t \mapsto e^{-i t H}$ for this system? It is a non-self-adjoint Hamiltonian,

$$
H=A+i B
$$


where $A$ and $B$ are self-adjoint operators each with a positive spectrum. The dynamics fails to be unitary because it is a non-isolated system that subtly interacts with its environment. The operators $A$ and $B$ do not necessarily commute, and so the Hamiltonian $H$ is not generally normal. It is also non-symmetric, and has a nonreal eigenvalue $E=E_{0}+i \Gamma$ by construction. So, the Hamiltonian for Gamow's quantum tunnelling system is one that fails all the criteria for observables that we have discussed so far. This example was discussed in the famous textbook by Landau and Lifshitz (1977, pgs. 555-556); and it has given rise to literatures that use nonself-adjoint Hamiltonians to describe quantum resonance and quantum optics (see e.g. Moiseyev 2011).

Although there are many other operators that are non-symmetric, non-normal, and have a non-real spectrum, it is not always easy to assign them a physical interpretation. In particular, if $A=B+i C$ and $B$ and $C$ do not commute, then there is no way to view $B$ and $C$ as jointly measurable observables. As an example of this, if $\sigma_{x}, \sigma_{y}, \sigma_{z}$ are the standard Pauli spin matrices, then we can formally write down the operator,

$$
R=\sigma_{x}+i\left(\sigma_{y}+\sigma_{z}\right)
$$

This operator has complex eigenvalues $\pm i$, associated with eigenvectors $\left(\begin{array}{l}i \\ 1\end{array}\right)$ and $\left(\begin{array}{l}0 \\ 1\end{array}\right)$, respectively. It is also non-symmetric and non-normal. Unfortunately, the physical significance of such an operator is also far from clear. It certainly does not admit an obvious interpretation as the generator of a dynamics.

These are just a few examples from the wilderness of non-self-adjoint operators. Much remains to be learned about the structural properties of such operators, such as their spectral theory and physical applications, and research in this area is ongoing. But this should not prevent us from exploring their possible use as observables. 


\section{Conclusion}

In this paper we have sorted non-self-adjoint operators into four classes: those that are normal, those that are symmetric or POVMs, those that have a real spectrum, and those that admit none of these properties. In spite of a pervasive dogma, nonself-adjoint operators may provide conceptual clarity or calculational convenience in modelling quantum systems. We have seen that the first class, that of normal operators, can individually be treated like standard quantum observables, although a set of them must in general be restricted to be a sharp set. In contrast, the second and third classes introduce varying amounts of new physics into the discussion, from time observables to new interaction Hamiltonians. The fourth class is a wilderness,

with many unknowns. But some of them can be used to fruitfully model quantum systems.

In his textbook on linear operators, E. Brian Davies gave an apt characterisation of the state of non-self-adjoint operators from a mathematical perspective:

Studying non-self-adjoint operators is like being a vet rather than a doctor: one has to acquire a much wider range of knowledge, and to accept that one cannot expect to have as high a rate of success when confronted with particular cases. (Davies 2007, p.x)

So too is the proposal to allow observables that are not self-adjoint. When an arbitrary non-self-adjoint operator from the great wilderness of possibilities is proposed as an observable, there may well be little that we can say about how to associate it with real-world observations. However, a number of interesting cases are well-understood, philosophically well-motivated, and lead to physically relevant models of quantum theory. It would be a pity if mere dogma prevented us from enjoying them.

\section{REFERENCES}

Aharonov, Y. and Bohm, D. (1961). Time in the quantum theory and the uncertainty relation for time and energy, Physical Review 122(5): 1649. 
Albert, D. Z. (1992). Quantum mechanics and experience, Harvard University Press. Bargmann, V. (1954). On unitary ray representations of continuous groups, Annals of Mathematics 59(1): 1-46.

Bender, C. (2007). Making sense of non-Hermitian Hamiltonians, Reports on Progress in Physics 70: 947-1018. https://arxiv.org/abs/hep-th/0703096.

Bender, C. M. and Boettcher, S. (1998). Real Spectra in Non-Hermitian Hamiltonians Having PT Symmetry, Physical Review Letters 80(24): 5243-5246. https: //arxiv.org/abs/physics/9712001.

Bender, C. M., Boettcher, S. and Savage, V. M. (2000). Conjecture on the interlacing of zeros in complex Sturm-Liouville problems, Journal of Mathematical Physics 41(9): 6381-6387. https://arxiv.org/abs/math-ph/0005012.

Bender, C. M., Brody, D. C. and Jones, H. F. (2002). Complex extension of quantum mechanics, Physical Review Letters 89(27): 270401.

Bender, C. M., Brody, D. C. and Jones, H. F. (2003). Must a Hamiltonian be Hermitian? arXiv: https://arxiv.org/abs/hep-th/0303005.

Berberian, S. K. (1966). Notes on Spectral Theory, Princeton: D. Van Nostrand Company Inc.

Blank, J., Exner, P. and Havlíček, M. (2008). Hilbert Space Operators in Quantum Physics, 2nd edn, Springer Science and Business Media B.V.

Born, M. and Heisenberg, W. (2009). Quantum mechanics (report for the 1927 Solvay Conference), in G. Bacciagaluppi and A. Valentini (eds), Quantum theory at the crossroads: Reconsidering the 1927 Solvay Conference, Cambridge: Cambridge University Press, pp. 372-405.

Born, M. and Jordan, P. (1925). Zur quantenmechanik, Zeitschrift für Physik 34(1): 858-888.

Busch, P. (2003). Quantum states and generalized observables: A simple proof of Gleason's theorem, Physical Review Letters 91(12): 120403. https://arxiv.org/ abs/quant-ph/9909073. 
Busch, P., Grabowski, M. and Lahti, P. J. (1994). Time observables in quantum theory, Physics Letters A 191(5-6): 357 - 361.

Busch, P., Grabowski, M. and Lahti, P. J. (1995a). Operational Quantum Physics, Berlin and Heidelberg: Springer-Verlag.

Busch, P., Grabowski, M. and Lahti, P. J. (1995b). Who Is Afraid of POV Measures? Unified Approach to Quantum Phase Observables, Annals of Physics 237(1): 1 11.

Butterfield, J. (2013). On Time in Quantum Physics, in H. Dyke and A. Bardon (eds), A Companion to the Philosophy of Time, Blackwell Companions to Philosophy, John Wiley and Sons, Inc., pp. 220-241. http://philsci-archive.pitt.edu/ 9287.

Cardano, G. (1968). Ars Magna, or, The Rules of Algebra, New York: Dover Publications, Inc. Originally published in 1545. Translated and Edited by T. Richard Witmer.

Conway, J. B. (1990). A Course in Functional Analysis, Vol. 96 of Graduate Texts in Mathematics, 2nd edn, Springer-Verlag New York, Inc.

Cooper, J. L. B. (1947). One-Parameter Semigroups of Isometric Operators in Hilbert Space, Annals of Mathematics 48(4): 827-842.

Cooper, J. L. B. (1948). Symmetric operators in Hilbert space, Proceedings of the London Mathematical Society 2(1): 11-55.

Davies, E. B. (2007). Linear operators and their spectra, Vol. 106 of Cambridge studies in advanced mathematics, Cambridge:Cambridge University Press.

Dirac, P. A. M. (1935). The Principles of 'Quantum' Mechanics, 2nd edn, Oxford: The Clarendon Press.

Dirac, P. A. M. (1947). The Principles of Quantum Mechanics, third edition edn, Oxford: Clarendon Press.

Dorey, P., Dunning, C. and Tateo, R. (2001a). Spectral equivalences, Bethe ansatz equations, and reality properties in PT-symmetric quantum mechanics, Journal 
of Physics A: Mathematical and General 34(28): 5679. https://arxiv.org/abs/ hep-th/0103051.

Dorey, P., Dunning, C. and Tateo, R. (2001b). Supersymmetry and the spontaneous breakdown of PT symmetry, Journal of Physics A: Mathematical and General 34(28): L391. https://arxiv.org/abs/hep-th/0104119.

Dubin, D. and Hennings, M. (1990). Quantum mechanics, algebras and distributions, Essex, UK: Longman Group UK Limited.

Duncan, A. and Janssen, M. (2009). From canonical transformations to transformation theory, 1926-1927: The road to Jordan's Neue Begründung, Studies in History and Philosophy of Modern Physics 40(4): 352-362.

Duncan, A. and Janssen, M. (2013). (Never) Mind your p's and q's: Von Neumann versus Jordan on the foundations of quantum theory, The European Physical Journal H 38(2): 175-259. http://philsci-archive.pitt.edu/9283/.

Earman, J. (2009). Essential self-adjointness: implications for determinism and the classical-quantum correspondence, Synthese 169(1): 27-50.

Euler, L. (1822). Elements of Algebra, London: Longman, Hurst, Rees, Orme, and Co. Edited by Rev. John Hewlett. Originally published in 1765.

Ewald, P. P. (1960). Session I, Interview of Max Born on 1 June 1960. Niels Bohr Library and Archives, American Institute of Physics, College Park, MD, http: //www . aip.org/history-programs/niels-bohr-library/oral-histories/4522-1.

Galapon, E. A. (2009). Post-Pauli's Theorem Emerging Perspective on Time in Quantum Mechanics, in G. Muga, A. Ruschhaupt and A. del Campo (eds), Time in Quantum Mechanics - Vol. 2, Lecture Notes in Physics 789, Springer-Verlag Berlin Heidelberg, pp. 25-63.

Gamow, G. (1928). Zur quantentheorie des atomkernes, Zeitschrift für Physik 51(34): $204-212$.

Griffiths, D. J. (1995). Introduction to Quantum Mechanics, Upper Saddle River, NJ: Prentice Hall. 
Hegerfeldt, G. C. and Muga, J. G. (2010). Symmetries and time operators, Journal of Physics A: Mathematical and Theoretical 43: 505303. arXiv:1008.4731.

Hermite, C. (1855). Remarque sur un théorème de M. Cauchy, Comptes rendus hebdomadaires des séances de l'Académie des sciences 41: 181-183.

Hilbert, D., von Neumann, J. and Nordheim, L. (1928). Über die Grundlagen der Quantenmechanik, Mathematische Annalen 98(1): 1-30.

Holevo, A. S. (1982). Probabilistic and Statistical Aspects of Quantum Theory, NorthHolland Series in Statistics and Probability Vol. 1, Amsterdam: North-Holland Publishing Co. Originally published in Russian in 1982.

Hughes, R. I. G. (1992). The structure and interpretation of quantum mechanics, Cambridge, MA: Harvard University Press.

Jammer, M. (1996). The conceptual development of quantum mechanics, New York, St. Louis, San Francisco, Toronto, London, Sydney: McGraw Hill Book Company. Jauch, J. M. and Piron, C. (1976). Generalized localizability, Helvetica Physica Acta 40.

Jordan, P. (1927a). Über eine neue Begründung der Quantenmechanik, Zeitschrift für Physik 40: 809-838.

Jordan, P. (1927b). Über eine neue Begründung der Quantenmechanik. II., Zeitschrift für Physik 44(1-2): 1-25.

Landau, L. D. and Lifshitz, E. M. (1977). Quantum mechanics: non-relativistic theory, Vol. 3 of Course of Theoretical Physics, 3rd edn, Oxford: Pergamon Press Ltd. Translated by J. B. Sykes and J. S. Bell.

Landsman, K. (2017). Foundations of Quantum Theory: From Classical Concepts to Operator Algebras, Cham, Switzerland: Springer International Publishing AG. Published Open Access: https://link.springer.com/book/10.1007/ 978-3-319-51777-3.

Lévai, G. and Znojil, M. (2001). Conditions for complex spectra in a class of PTsymmetric potentials, Modern Physics Letters A 16(30): 1973-1981. https:// 
arxiv.org/abs/quant-ph/0110064.

Lévy-Leblond, J.-M. (1976). Who is afraid of nonhermitian operators? A quantum description of angle and phase, Annals of Physics 101(1): 319 - 341.

London, F. (1926). Über die Jacobischen Transformationen der Quantenmechanik, Zeitschrift für Physik 37(12): 915-925.

Ludwig, G. (1971). The measuring process and an axiomatic foundation of quantum mechanics, in B. d'Espagnat (ed.), Foundations of Quantum Mechanics: Proceedings of the International School of Physics 'Enrico Fermi', Course IL, Varenna on Lake Como, Villa Monastero, New York: Academic Press.

Ludwig, G. (1983). Foundations of Quantum Mechanics, Vol. I, Springer-Verlag New York, Inc. Translated by Carl A. Hein from the 1954 German edition.

Messiah, A. (1999). Quantum Mechanics, Two Volumes Bound as One, New York: Dover.

Moiseyev, N. (2011). Non-Hermitian quantum mechanics, Cambridge University Press.

Muga, J. G., Sala Mayato, R. and Equisquiza, Í. L. (2008). Introduction, in J. G. Muga, R. Sala Mayato and Í. L. Equisquiza (eds), Time in Quantum Mechanics - Vol.1, second edn, Vol. 734 of Lecture notes in physics, Springer-Verlag Berlin Heidelberg, pp. 1-30.

Naimark, M. A. (1940). Self-adjoint extensions of the second kind of a symmetric operator, Izvestiya Rossiiskoi Akademii Nauk. Seriya Matematicheskaya 4(1): 53104. Originally published as 'O samosoprjažennyh rasširenijah vtorogo roda simmetričeskogo operatora' by the Steklov Mathematical Institute, Russian Academy of Sciences.

Naimark, M. A. (1968). Linear differential operators, London:Frederick Ungar Publishing Co., Inc. Parts I and II, Translated by E. R. Dawson and edited by W. N. Everitt. 
Pashby, T. (2014). Time and the foundations of quantum mechanics, PhD thesis, University of Pittsburgh. http://philsci-archive.pitt.edu/10666/.

Pauli, W. (1980). General Principles of Quantum Mechanics, Springer-Verlag: Berlin Heidelberg New York.

Penrose, R. (2004). The Road to Reality: A Complete Guide to the Laws of the Universe, London: Jonathan Cape.

Reed, M. and Simon, B. (1975). Methods of Modern Mathematical Physics II: Fourier Analysis, Self-Adjointness, San Diego: Academic Press, Inc.

Reichenbach, H. (1944). Philosophical Foundations of Quantum Mechanics, Berkeley and Los Angeles: University of California Press.

Roberts, B. W. (2017). Unreal observables, Philosophy of Science 84(5): 1265-1274. http://philsci-archive.pitt.edu/12965/.

Rudin, W. (1991). Functional Analysis, 2nd edn, McGraw-Hill, Inc.

Sakurai, J. J. (1994). Modern Quantum Mechanics, revised edn, Reading, MA: Addison Wesley.

Srinivas, M. and Vijayalakshmi, R. (1981). The 'time of occurrence' in quantum mechanics, Pramana 16(3): 173-199.

Streater, R. F. (2007). Lost Causes in and beyond Physics, Springer-Verlag Berlin Heidelberg.

Uhlhorn, U. (1963). Representation of symmetry transformations in quantum mechanics, Arkiv för Fysik 23: 307-340.

Weigert, S. (2003). Completeness and orthonormality in PT-symmetric quantum systems, Physical Review A 68(6): 062111. https://arxiv.org/abs/quant-ph/ 0306040v1.

Weinberg, S. (2013). Lectures on quantum mechanics, Cambridge: Cambridge University Press. 
Wüthrich, C. (2011). Can the world be shown to be indeterministic after all?, in C. Beisbart and S. Hartmann (eds), Probabilities in Physics, Oxford: Oxford University Press. http://philsci-archive.pitt.edu/8437/.

Znojil, M. (2015). Non-self-adjoint operators in quantum physics: Ideas, people, and trends, in F. Bagarello, J. Gazeau, F. Szafraniec and M. Znojil (eds), Nonselfadjoint operators in quantum physics: Mathematical aspects, Hoboken, New Jersey: John Wiley and Sons, Inc., pp. 7-58. 